\title{
Sgr.A* effect on Mercury and Trans-Neptune Objects
}

\author{
J.C. Hodge $\mathrm{e}^{1 *}$ \\ ${ }^{1}$ Retired, 477 Mincey Rd., Franklin, NC, 28734
}

\begin{abstract}
The Scalar Theory of Everything (STOE) has suggested the plenum from the Source of the Milky-Way galaxy (Galaxy) causes the observed rotation curves of galaxies and is the cause of the "Planet 9" orbits of icy objects. A correlation of the apsidal lines of the rocky planets is also consistent with their being a plenum caused force influencing the orbits. The study of planetary objects should include a parameter reflecting the effect of the STOE Source.
\end{abstract}

The orbit of Mercury is eccentric and inclined relative to other planets. The orbits of Trans-Neptune Objects (TNOs) have also been measured to be eccentric and highly inclined to the plane of the solar system. The Scalar Theory Of Everything proposed a new physics explanation of the TNOs to replace the "Planet 9" traditional explanation (Hodge 2019). This explanation was predicted by the explanation of the Pioneer Anomaly (Hodge 2006)

(Bartlett 2020) suggested the perihelion of Mercury resembles the orbits of the TNOs. Therefore, the orbit of Mercury should have a relation to the Scalar Theory of Everything (STOE) Source (Sagittarius A*). This is surprising for the STOE because the inner planets are rocky objects and the TNOs are icy-with-methane objects. The STOE suggests the divergence of the plenum (hence force) from the Source at Sagittarius A* (Sgr A*) acts on the surface area (a radius ${ }^{-2}$ relation). Gravity acts on the mass (radius ${ }^{-3}$ relation). The difference causes the decreasing metallicity with increasing Galactic radius relation, pushing lighter elements outward.

\footnotetext{
*E-mail: jchodge@frontier.com
} 
This paper examines the data.

Table 1 Shows the aphelion (Ap.)and perihelion (Peri.) of the rocky planets and two of the more extreme TNOs. The two TNOs listed represent the RA extremes.

The last line shows the position of Sgr A* in the Ap. column. The Source pushes matter from it to the coordinates in the Peri. column. Therefore, the Source effect is to cause a perihelion on objects in the listed perihelion direction.

A tendency of solar system objects is to align their apsidal lines and, also, their orbital planes with the STOE plenum force. Mercury is the closest aligned of the objects listed. Venus and Earth also show a tendency to align. Mars seems the outlier.

The solar system is revolving around the Galaxy center. This causes a change in the relative direction of the Source force of $\approx 0.014^{\circ} /$ year. This change in direction could cause the objects to deviate inclination of the plane of the solar system. Such change would be more pronounced in icy objects than in rocky objects. This is measured in TNOs. Those objects with very long rotation periods would experience a greater changing direction of the force. This would present a very confusing orbit to gravitational only orbit calculations as some of the simulations show.

This further suggests the TNOs are being pushed out of the solar system on orbits becoming hyperbolic.

This force would also lift the perihelia of long-period Kuiper Belt Objects (KBOs) as their orbit data shows that explain the origin of the Neptunedetached KBOs. At the same time, Oort cloud objects traveling toward Sgr $A^{*}$ have their orbits reduced. This, also, could be a source of long-period KBOs.

Because the TNOs are icy objects and they venture far from the gravity of the Sun, the effect of the STOE Source is more pronounced. Perhaps, the simulations of the TNOs orbits would be more accurate with consideration of the Source.

The experience of the long, fruitless search for Dark Matter and the search for Vulcan suggest new physics should be considered.

The study of planetary objects should include a parameter reflecting the effect of the STOE Source. Perhaps one of the PPN factors should be this force. 
Table 1: Aphelion and perihelion of some solar system objects. ${ }^{\text {a }}$

\begin{tabular}{|c|c|c|c|c|c|c|}
\hline Object & $\begin{array}{l}\text { Ap. } \\
\text { RA } \\
\text { hours }\end{array}$ & $\begin{array}{l}\text { DEC } \\
\text { deg. }\end{array}$ & $\begin{array}{l}\text { Dist. }^{c} \\
\text { AU }\end{array}$ & $\begin{array}{l}\text { Peri. }^{b} \\
\text { RA } \\
\text { hours }\end{array}$ & $\begin{array}{l}\text { DEC } \\
\text { deg. }\end{array}$ & $\begin{array}{l}\text { Dist. }^{c} \\
\text { AU }\end{array}$ \\
\hline Mercury $^{\mathrm{d}}$ & $17^{\mathrm{h}} 03^{\mathrm{m}}$ & $-26^{\mathrm{o}} 14^{\mathrm{m}}$ & 0.47 & $05^{\mathrm{h}} 03^{\mathrm{m}}$ & $+26^{\mathrm{o}} 14^{\mathrm{m}}$ & 0.31 \\
\hline Venus $^{\mathrm{e}}$ & $20^{\mathrm{h}} 58^{\mathrm{m}}$ & $-20^{\mathrm{o}} 02^{\mathrm{m}}$ & 0.73 & $08^{\mathrm{h}} 59^{\mathrm{m}}$ & $+19^{\mathrm{o}} 58^{\mathrm{m}}$ & 0.72 \\
\hline $\operatorname{Earth}^{\mathrm{f}}$ & $18^{\mathrm{h}} 51^{\mathrm{m}}$ & $-22^{\mathrm{o}} 55^{\mathrm{m}}$ & 1.02 & $07^{\mathrm{h}} 02^{\mathrm{m}}$ & $+22^{\mathrm{o}} 41^{\mathrm{m}}$ & 0.98 \\
\hline Mars $^{g}$ & $10^{\mathrm{h}} 34^{\mathrm{m}}$ & $+10^{\mathrm{o}} 54^{\mathrm{m}}$ & 1.67 & $22^{\mathrm{h}} 34^{\mathrm{m}}$ & $-10^{\mathrm{o}} 53^{\mathrm{m}}$ & 1.38 \\
\hline TNOs & & & & & & \\
\hline Sedna ${ }^{h}$ & & $?$ & & $06^{\mathrm{h}} 27^{\mathrm{m}}$ & $+14^{\mathrm{o}} 23^{\mathrm{m}}$ & 76.2 \\
\hline $2007 \mathrm{TG} 422^{\mathrm{i}}$ & & $?$ & & $02^{\mathrm{h}} 51^{\mathrm{m}}$ & $-02^{\mathrm{o}} 21^{\mathrm{m}}$ & 35.6 \\
\hline Sgr.A* j & $17^{\mathrm{h}} 46^{\mathrm{m}}$ & $-29^{\circ} 00^{\mathrm{m}}$ & $1.6 \times 10^{8}$ & $05^{\mathrm{h}} 46^{\mathrm{m}}$ & $+29^{\circ} 00^{\mathrm{m}}$ & \\
\hline
\end{tabular}

a Data for planets from https://ssd.jpl.nasa.gov/horizons.cgi . Coordinate system is Sun centered, equatorial.

b Dates for aphelion and perihelion from www.skycaramba.com/aphelions.shtml and www.skycaramba.com/perihelions.shtml .

c Distance from the center of the Sun.

d Dates: 7 Feb. 2017, 23 Mar. 2017.

e Dates: 12 Jun. 2017, 20 Feb. 2017.

f Dates: 3 Jul. 2017, 4 Jan. 2017.

g Dates: 07 Oct. 2017, 29 Oct. 2016.

h Dates: 14 Jul. 2076.

i Dates: 24 Oct. 2005.

j J2000 Wikipedia. 


\section{REFERENCES}

\section{References}

Bartlett, R., 2020, NON-COMPUTABILITY AND UNPREDICTABILITY ARE SO YESTERSDAY: WITH COMPUTABLE AND PREDICTABLE COSMIC STRUCTURE, PLUS IMPLICATIONS FOR MATHEMATICS AND SCIENCE, FQXi 2020 contest, https://fqxi.org/community/forum/topic/3382 .

Hodge, J.C., 2006. Scalar potential model of the Pioneer Anomaly, preprint http://arxiv.org/abs/astro-ph?0612567v1.pdf .

Hodge, J.C., 2019, STOE explains "Planet 9" , IntellectualArchive, Vol.8, No. 2, P.13, http://intellectualarchive.com/?link=item\&id=2089. 